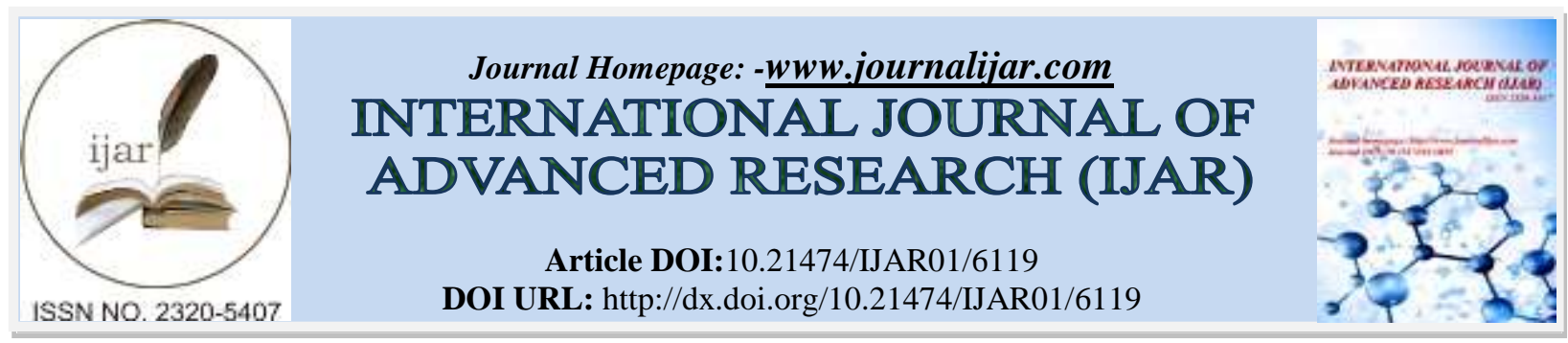

RESEARCH ARTICLE

\title{
CHRONIC PANCREATITIS RARE COMPLICATION, PANCREATIC-PLEURAL FISTULA \&PLEURAL PSEUDOCYST: A CASE REPORT.
}

Amal Salem ${ }^{1}$, Ahmed Azhari ${ }^{1}$, Amjad Banjar ${ }^{2}$ and Maher Ghazzawy ${ }^{3}$.

1. Medical Intern, Umm Al-Qura University, Makkah, Saudi Arabia.

2. Radiology Resident, the Saudi Board of Radiology, Makkah, Saudi Arabia.

3. Radiologist, Al-Noor Specialist Hospital, Makkah, Saudi Arabia.

\section{Manuscript Info}

\section{Manuscript History}

Received: 20 October 2017

Final Accepted: 22 November 2017

Published: December 2017

\section{Keywords:-}

Pancreatic-pleural fistula, Pleural pseudocyst, Pancreatitis complications, Amylase level, Pleural fluid.

\section{Abstract}

Background: A pancreatic-pleural fistula is a complication of a pancreatic pseudocyst due to cranial extension of an erosive tract that forms a communication between the pseudocyst and the pleural cavity with subsequent pleural effusion.

Case presentation: A 16-year-old boy presented with a history of shortness of breath and cough over 3 months, with diffuse abdominal pain radiating to his back. A chest X- ray showed massive left-sided pleural effusion without any mediastinal shift. Computed tomography of the chest and abdomen showed a pancreatic-pleural fistula. This diagnosis was confirmed after thoracentesis performed for pleural fluid sampling showed an amylase level of 28,459 IU/L (reference range 30$100 \mathrm{IU} / \mathrm{L})$. He was treated medically and an endoscopic retrograde cholangiopancreatography was performed.

Conclusion: A pancreatic-pleural fistula is a rare complication of chronic pancreatitis, and endoscopic surgery is a useful treatment option in those who fail medical treatment, fluid drainage, and/or fistula stent.

Copy Right, IJAR, 2017,. All rights reserved. 


\section{Introduction:-}

Pancreatic pseudocysts often complicate chronic pancreatitis. Leakage of pancreatic enzymes triggers fistula formation due to erosion of the bowel wall into a hollow viscus, which communicates with other cavities.

Fistula formation between a pancreatic pseudocyst and the pleura is rare and could present with a pleural effusionsuch effusions typically show high amylase content [J. L. Cameron.1978] because of direct drainage of pancreatic secretions into the pleural cavity secondary to acute/chronic inflammation and trauma/iatrogenic injury to the pancreatic duct. We describe a patient with chronic pancreatitis complicated by pancreatic-pleural fistula (PPF) formation with pleural effusion and pseudocyst.[Sommer CA. 2014]

\section{Case History:-}

A 16-year-old boy presented to the hospital with progressive shortness of breath and cough over 3 months, with diffuse abdominal pain radiating to his back and left shoulder. Eating exacerbated his pain, which was associated with nausea and vomiting. He reported positive history of weight loss and loss of appetite, but no fever, jaundice, itching, night sweats, melaena or hematemesis.

Upon admission, his temperature was $37.1^{\circ} \mathrm{C}$, blood pressure was $120 / 70$, pulse rate was $120 / \mathrm{min}$, respiratory rate was $22 / \mathrm{min}$, and oxygen saturation was $95 \%$ on room air.

Chest examination revealed left-sided decreased air entry with stony dullness to percussion and right-sided tracheal deviation.

Laboratory investigations revealed a white blood cell count of $8.54 \mathrm{~K} / \mathrm{mm} 3$ (reference range $4-11 \mathrm{~K} / \mathrm{mm} 3$ ), hemoglobin of $14.8 \mathrm{~g} / \mathrm{dL}$ (reference range $13-18 \mathrm{~g} / \mathrm{dL}$ ), amylase of $161 \mathrm{IU} / \mathrm{L}$ (reference range $25-115 \mathrm{IU} / \mathrm{L}$ ) with normal electrolytes, and liver function tests.

A chest X-ray showed massive left-sided pleural effusion, as Figure 1.

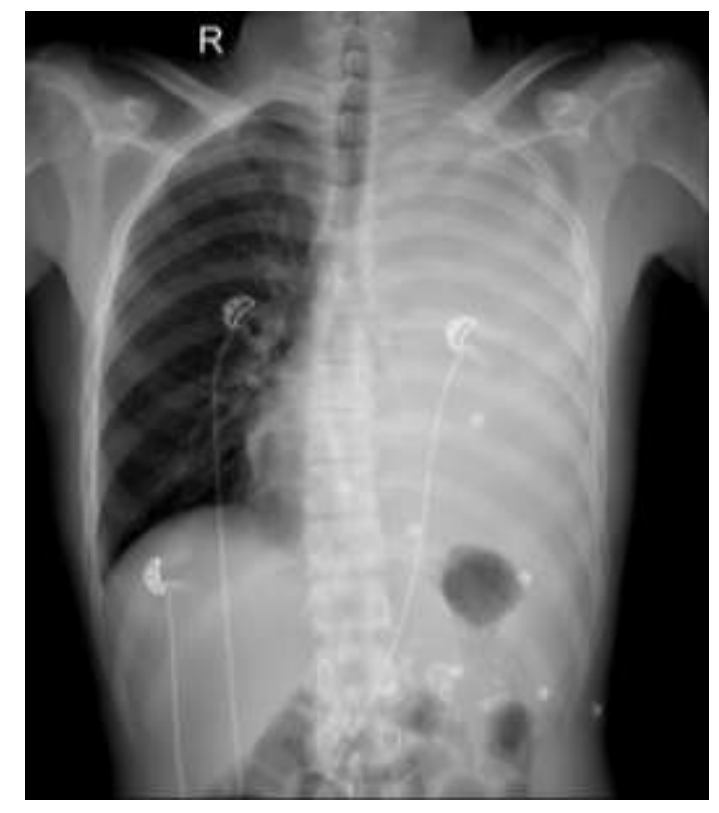

Figure 1:- Erect PA Chest X-

ray shows : complete

opacification of the left hemithorax, no mediastinal shift, with multiple variable sized calcification are noted in upper abdomen.

Computed tomography (CT) of the chest and abdomen showed:

\section{Chest:-}

A large pleural effusion occupying the entire left hemithorax with peripheral smooth enhancement of the pleura and no calcification. A small soft tissue density is noted in the dependent portion of the left lung base, likely representing debris. The left lung shows complete collapse. A small ill-defined enhanced exophytic nodule-like thickening is visualised within the collapsed parenchyma, likely related to the collapsed lung. Another small ill-defined collection 
is noted perivascularly in the upper mediastinum showing smooth peripheral enhancement. The medial aspect of the right lower lobe shows small ill-defined consolidation, likely secondary to compression of the displaced adjacent mediastinal structures. No other nodules, masses, cavitary lesions, or pleural effusion are noted.

\section{Abdomen and pelvis:-}

Multiple, variable-sized and shaped intra-ductal pancreatic calcifications are noted - the largest measuring $2 \times 1.2$ $\mathrm{cm}$ in the intra-ductal portion of the body. The pancreas is small in size. A tubular tract (likely fistula) is observed originating from the distal pancreatic body/tail, ascending toward the crus of the left hemidiaphragm. The adjacent major vascular structures are grossly intact, as Figure 2 .
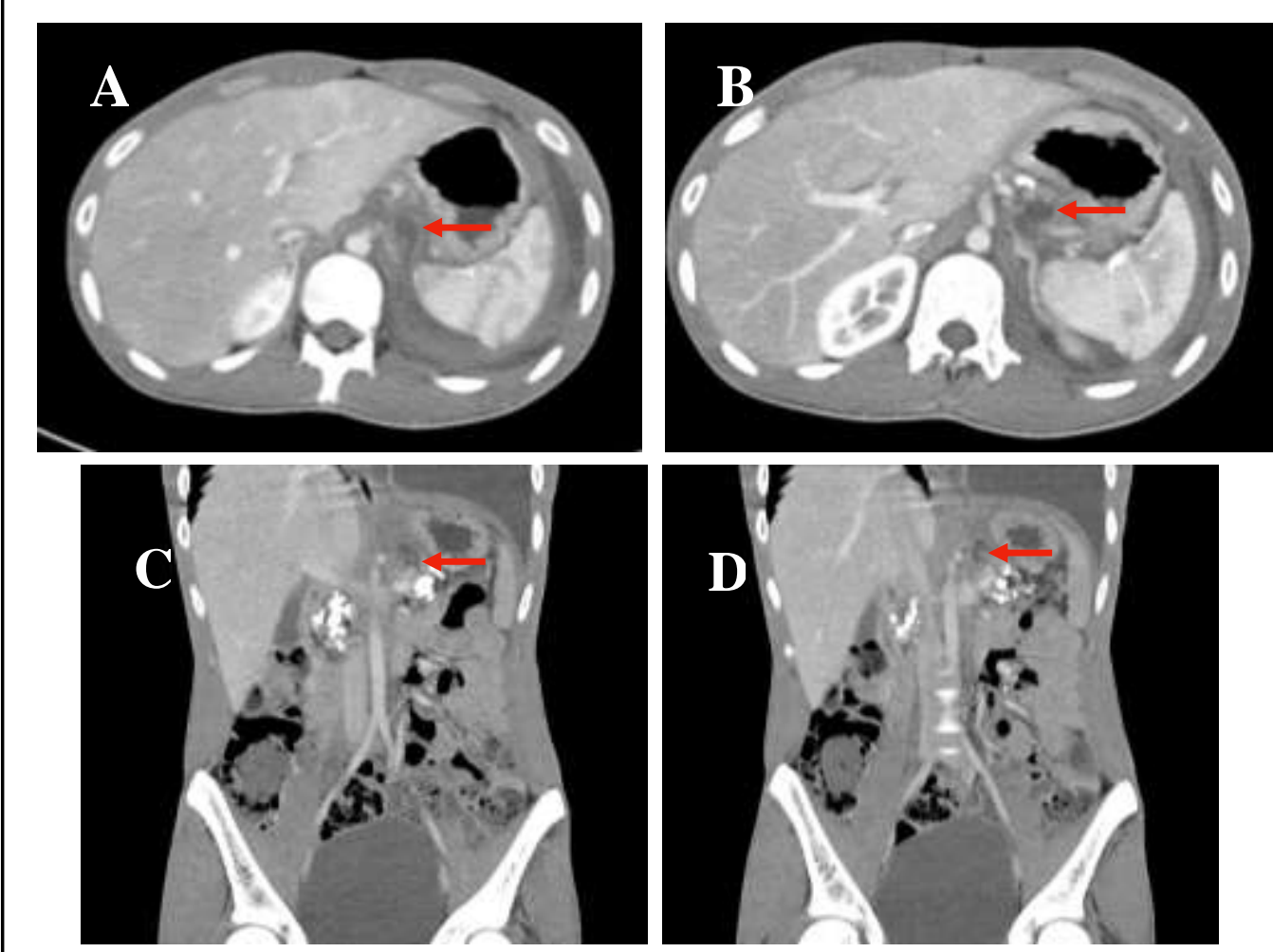

Figure 2:- (A) (B) axial, (C) (D) coronal computed tomography (CT) images with IV contrast show : tubular tract arising from the distal body/tail of the pancreas ascending upward toward the crus of the left hemidiaphragm, likely representing a fistulous tract .

The patient was administered broad-spectrum antibiotics, and analgesics and underwent left-sided thoracentesis with drainage of $1800 \mathrm{~cm} 3$ of pleural fluid, which showed an amylase level of 28,459 IU/L (reference range 30-100 $\mathrm{IU} / \mathrm{L})$.

On the second day of hospitalization, he underwent magnetic resonance cholangiopancreatography (MRCP) for diagnostic confirmation of the pancreatic fistula, and we observed atrophic pancreas with a dilated pancreatic duct and multiple pancreatic duct stones. A $1.6 \mathrm{~cm}$ defect noted in the distal body of the pancreas likely represented the site of leakage, as Figure 3 . 

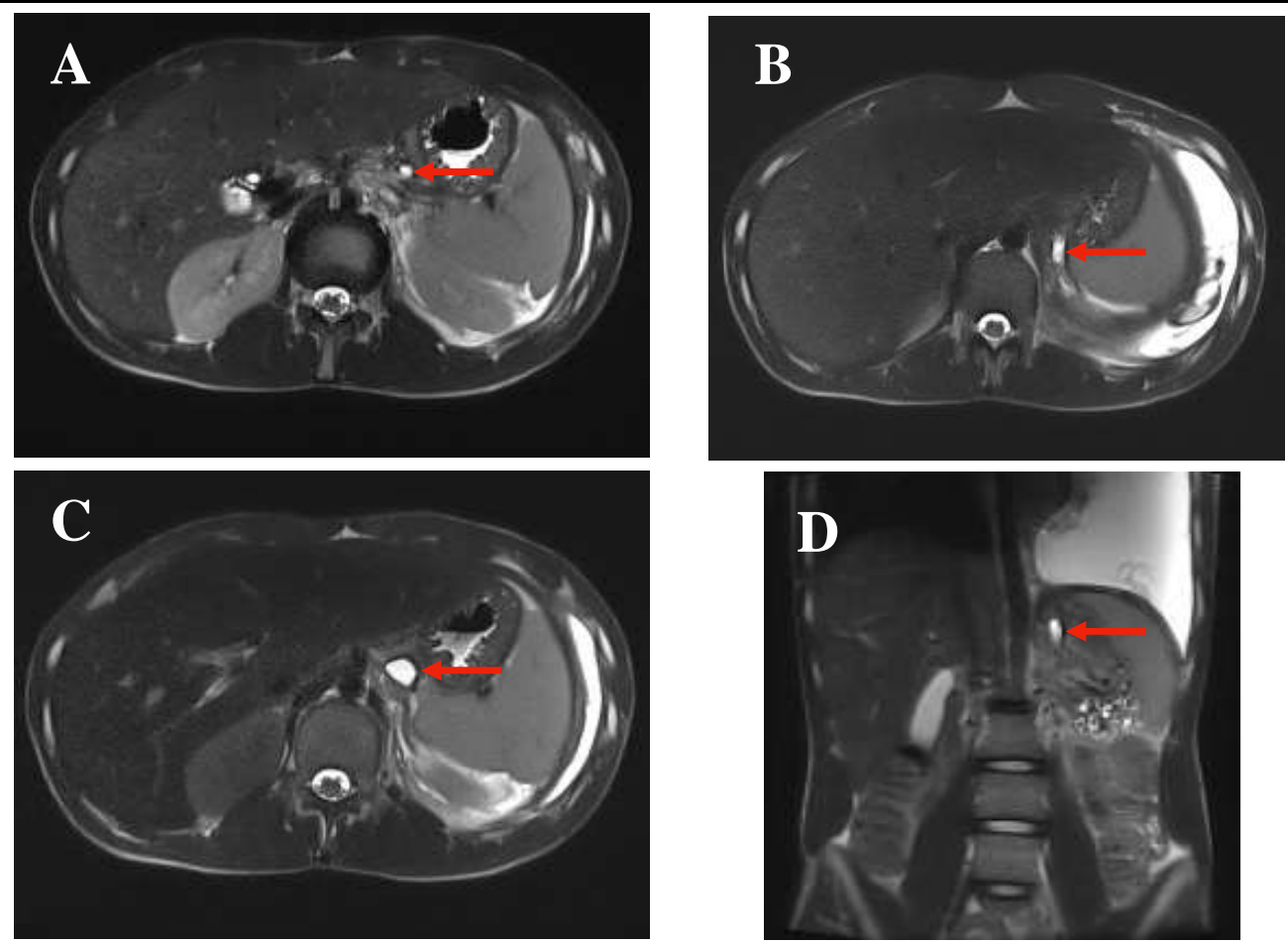

Figure 3 : Axial view in figures (A-B-C), Coronal view in figure (D) of Magnetic resonance cholangiopancreatography (MRCP)T2 HASTE show : oblong, vertically-oriented, peri-pancreatic tract that extends to the left sub-phrenic space, anterior to the left adrenal gland and ends below the left hemidiaphragm.

On day 4, he underwent endoscopic retrograde cholangiopancreatography (ERCP) to visualise the fistulous tract, and a 7 French stent was successfully inserted with good drainage.

The patient's symptoms improved post procedure, and he was discharged from the hospital on day 8 but was lost to follow-up.

\section{Discussion:-}

Acute and/or chronic pancreatitis is associated with the accumulation of enzyme-rich fluid, which after 6 weeks, gets encapsulated by granulation tissue and extracellular matrix to form pancreatic pseudocysts without an epithelial lining unlike true cysts.[Lerch MM. 2009 ,Habashi S. 2009] Pseudocysts occur in 5-16\% of patients,[Aghdassi AA. 2006] and 0.4-4.5\% of patients develop PPF.[Lamme B. 2003 ,Uchiyama T. 1992]

The index of clinical suspicion is high in those with a history of upper abdominal trauma, surgery, alcoholism, or prior hospitalisation for pancreatitis.[H. Xu. 2013] Trans-abdominal ultrasonographic examination distinguishes between different types of cysts, and CT is a highly sensitive (82-100\%) diagnostic tool[Ajmera AV. 2012 ,Imai Y. 1993] to identify lesions that could get complicated particularly in patients with internal fistulas. Magnetic resonance cholangiopancreatography (MRCP) enables visualisation of pancreatic ducts,[S. Vyas. 2009] pseudocysts, and also fistulous tracts. Multiple modalities were used in our patient-an initial chest X-ray showed a massive leftsided pleural effusion associatedwith multiple variable-sized calcifications in the upper abdominal area corresponding to the pancreasindicatingchronicpancreatitis. Subsequent CT examinationrevealeda large pseudocyst in the left pleural cavity and a tubular tract suggestingfistula formation between the pancreaticpseudocyst and the pleural cavity - a finding confirmed by an MRCP. Thoracentesis was performed, and pleural fluid analysis showed a high amylase level of 28,459 IU/L, which confirmed the diagnosis.

PPF shows a variable presentation based on the size and the location of the fistula. Patients with a PPF present with dyspnoea, abdominal pain, cough, and/or chest pain (observed in $68 \%$ of patients).[Everton C. 2017] Our patient 
showed dyspnoea, cough and diffuse abdominal pain radiating to the back and left shoulder associated with nausea and vomiting.

Pancreatic pseudocysts occur secondary to leakage of enzymes that damage the pancreatic duct to form fluid collections that are typically confined to the lesser sac, although these can extend cranially or caudally along preferential drainage pathways, such as the lesser sac, the anterior/posterior para-renal space, the peri-splenic area, and the lower lobe of the liver.[H. Xu. 2013, S. S. Siegelman. 1980] In patients showing thoracic involvement, posterior disruption causes leakage of pancreatic enzymes into the retroperitoneal space[J. L. Cameron. 1978,T.Yasuda. 2007, R. Gupta. 2007] and mediastinal tracking transdiaphragmatically or via the aortic/esophageal hiatus.[J. L. Cameron. 1978,T.Yasuda. 2007, R. Gupta. 2007, C. Panackel. 2008 ]

Patients with ascending pancreatitis showing pleural effusion present with two types of lesions [K. Wypych. 2011] : (1) Those associated with acute pancreatitis are usually small, sympathetic in nature, primarily left-sided, with a normal amylase $(<100 \mathrm{U} / \mathrm{L})$ and a low protein content. These are self-limited and require no treatment.[R. Gupta. 2007, K. Wypych. 2011,S. Gu tz. 2004] (2) Those associated with a PPF (as was observed in our patient) show a direct communication with the pleura, are large-sized, unilateral, recurrent, with a high amylase (> 1000U/L) and protein (> $33 \mathrm{~g} / \mathrm{dL}$ ) content.[J. L. Cameron. 1978]

Several treatment strategies are used to manage PPF including medical treatment, drainage of the fluid collection, or endoscopic surgery, which shows lower morbidity rates than those noted with open surgery.[Lerch MM. 2009] Treatment is optimised based on symptom severity, anatomy of the fistulous tract, size of the fluid collection, and the available expertise. Our patient was treated medically with broad-spectrum antibiotics and analgesics, followed by thoracentesis for drainage of the fluid. An ERCP was performed to visualise the fistulous tract, and a 7 French stent was inserted.

\section{Conclusion:-}

Although pancreatic pseudocyst formation is a known complication of chronic pancreatitis, a PPF presenting with a pleural effusion is rare. An MRCP is the gold standard diagnostic test, and pleural fluid obtained via diagnostic thoracentesis reveals high amylase levels $(>1000 \mathrm{U} / \mathrm{L})$. The management strategy comprises initial medical treatment, followed by fluid drainage, and ERCP-guided stent placement into the fistulous tract. In refractory cases, endoscopic surgery is required.

\section{Acknowledgements:-}

Authors are thankful to Radiology department, Alnoor Specialist Hospital, Makkah, Saudi Arabia for providing the radiological images to carry out this case report.

\section{References:-}

1. Aghdassi AA, Mayerle J, Kraft M.(2006): Pancreatic pseudocysts-when andhow to treat, HPB (Oxford)8:43241.

2. Ajmera AV, Judge TA. (2012): Mediastinal extension of pancreatic pseudocyst-a case with review of topic and management guidelines. Am J Ther. 19(5): e152-e156.

3. C. Panackel, A. T. Korah, D. Krishnadas, and K. R. Vinayaku- mar. (2008): Pancreatic pseudocyst presenting as dysphagia: a case report, Saudi Journal of Gastroenterology, 14(1):28-30.

4. Everton C, Marcio A, Martinho A. (2017): mangment of pancreaticopleural fistulas secondary to chronic pancreatitis. ABCD Arq Bras Cir Dig review Article. 30(3):225-228.

5. Habashi S, Draganov PV. (2009): Pancreatic pseudocyst. World J Gastroenterol

6. $15: 38-47$.

7. H. Xu, X. Zhang, A. Christe.(2013): Anatomic pathways of peripancreatic fluid draining to mediastinum in recurrent acute pancreatitis: Visible Human Project and CT Study, PLoS ONE, vol.8, no.4, Article ID e62025.

8. Imai Y, Taniguchi M, Tagawa K. (1993): A case of chronic pancreatitis complicated by massive pericardial and right pleural effusion. GastroenterolJpn, 28(5): 734-738.

9. J. L. Cameron. (1978): Chronic pancreatic ascites and pancreatic pleural effusions, Gastroenterology. 74(1):134-140.

10. K. Wypych, Z. Sera n, P. GaŁazka. (2011): Pancreaticopleural fistulas of different origin: report of two cases and a review of literature, Polish Journalof Radiology, 76(2):56-60. 
11. Lamme B, Boerma D, Boermeester MA, Gouma DJ. (2003): Pleural fluid in chronic pancreatitis (in Dutch). Ned Tijdschr Geneeskd147:1437-1441.

12. Lerch MM, Stier A, Wahnschaffe U.(2009): Pancreatic pseudocysts: observation, endoscopic drainage, or resection. DtschArzteblInt 106: 614-621.

13. R. Gupta, J. C. Munoz, P. Garg, G. Masri, N. S. Nahman Jr., and L. R. Lambiase. (2007): Mediastinal pancreatic pseudocyst - a case report and review of the literature,Medscape General Medicine, vol. 9, no. 2, article 8 .

14. S. Gu tz, H. Gosse, U. Busse, and A. Gillissen, (2004): Patient with left sided pleural effusion and large structures of so -tissue-density in the mediastinum. A rare case of acute pancreatitis with involvement of the pleural and mediastinalcompartiment, Pneumologie,58(10):724-727.

15. Sommer CA and Wilcox CM. (2014): Pancreatico-pericardial fistula as a complication of chronic pancreatitis [version 1; referees: 2 approved] F1000Research 3:31 (doi: 10.12688/f1000research.3-31.v1).

16. S. S. Siegelman, B. E. Copeland, G. P. Saba, J. L. Cameron, R. C. Sanders, and E. A. Zerhouni. (1980): CT of fluid collections associated with pancreatitis, American Journal of Roentgenology134(6):1121-1132.

17. S. Vyas, D. Gogoi, S. K. Sinha, P. Singh, T. D. Yadav, and N. Khandelwal. (2009): Pancreaticopleural fistula: an unusual complication of pancreatitis diagnosed with magnetic resonance cholangiopancreatography, Journal of the Pancreas, 10(6):671-673.

18. T.Yasuda,T.Ueda,Y.Fujinoetal. (2007): Pancreaticobronchial fistula associated with chronic pancreatitis: report of a case, Surgery Today. 37(4)338-341.

19. Uchiyama T, Suzuki T, Adachi A, Hiraki S, Iizuka N. (1992): Pancreatic pleural effusion: case report and review of 113 cases in Japan. Am J Gastroenterol 87:387-391. 